\title{
miRNA-26a-5p and miR-26b-5p inhibit the proliferation of bladder cancer cells by regulating PDCD10
}

\author{
KE WU $^{1 *}$, XING-YU MU ${ }^{*}$, JUN-TAO JIANG ${ }^{1}$, MING-YUE TAN $^{1}$, REN-JIE WANG ${ }^{1}$, WEN-JIE ZHOU ${ }^{2}$, \\ XIANG WANG ${ }^{1}$, YIN-YAN HE ${ }^{3}$, MING-QING LI ${ }^{2}$ and ZHI-HONG LIU ${ }^{1}$ \\ ${ }^{1}$ Department of Urology, Shanghai General Hospital, Shanghai Jiao Tong University School of Medicine, \\ Shanghai 200080; ${ }^{2}$ Laboratory for Reproductive Immunology, Hospital of Obstetrics and Gynecology, \\ Fudan University, Shanghai 200011; ${ }^{3}$ Department of Obstetrics and Gynecology, Shanghai General Hospital, \\ Shanghai Jiao Tong University School of Medicine, Shanghai 200080, P.R. China
}

Received October 11, 2017; Accepted September 4, 2018

DOI: $10.3892 / o r .2018 .6734$

\begin{abstract}
MicroRNA (miR)-26a-5p and miR-26b-5p consistently play an antitumor role in many types of cancers, but the underlying mechanism remains unclear in bladder cancer (BC). In the present study, we found that, in $\mathrm{BC}$ tissues, the levels of miR-26a-5p and miR-26b-5p were lower than in paired normal tissues. The upregulation of miR-26-5p significantly inhibited the proliferation of BC cell lines (T24 and 5637). Bioinformatics analysis indicated that Programmed Cell Death 10 (PDCD10) was the downstream target gene of miR-26a-5p/miR-26b-5p, and this was ascertained by western blotting and quantitative real-time reverse transcription PCR (RT-qPCR). In addition, in the 3'-UTR of PDCD10, the binding site was identified using a luciferase reporter assay. We determined that clinical BC tissues presented higher PDCD10 levels than adjacent normal tissues and that PDCD10 promoted proliferation of BC cell lines. Overexpression of $\mathrm{miR}-26 \mathrm{a}-5 \mathrm{p} / \mathrm{miR}-26 \mathrm{~b}-5 \mathrm{p}$ inhibited the stimulatory effect on proliferation of $\mathrm{BC}$ cells induced by PDCD10. In addition, in vivo experiments and clinical data revealed that the prognosis of $\mathrm{BC}$ patients with high expression of miR-26a-5p/miR-26b-5p and low expression of PDCD10 was better than that of patients with low miR-26-5p and high PDCD10 expression. These data revealed that miR-26a-5p
\end{abstract}

Correspondence to: Professor Zhi-Hong Liu, Department of Urology, Shanghai General Hospital, Shanghai Jiao Tong University School of Medicine, 100 Haining Road, Shanghai 200080, P.R. China

E-mail: drzhihongliu@sjtu.edu.cn

Professor Ming-Qing Li, Laboratory for Reproductive Immunology, Hospital of Obstetrics and Gynecology, Fudan University, 419 Fangxie Road, Shanghai 200011, P.R. China

E-mail:mqli@fudan.edu.cn

${ }^{*}$ Contributed equally

Key words: bladder cancer, miR-26a-5p, miR-26b-5p, PDCD10, proliferation and miR-26b-5p were pivotal regulators in $\mathrm{BC}$ progression by targeting the proliferation-related protein, PDCD10. The miR-26-5p/PDCD10 interaction may provide important insight into the pathway of $\mathrm{BC}$ progression and present novel opportunities for future diagnosis and treatment strategies, especially for patients with high levels of PDCD10.

\section{Introduction}

Bladder cancer (BC) is one of the most important urinary cancers and it remains a difficult cancer to treat due to metastasis and rapid recurrence (1). The characteristics of $\mathrm{BC}$ include rapid development and high invasion and metastasis potential. Once distant metastasis is present, $\sim 80 \%$ of $\mathrm{BC}$ patients will have succumbed to this disease in the first 5 years (2). Research has focused on studying the mechanisms of $\mathrm{BC}$, but little progress has been achieved. Therefore, the definitive mechanisms, as well as novel prognostic markers for $\mathrm{BC}$ need to be discovered.

Studies of RNA networks, especially new patterns of molecular interactions, are beneficial to the prognosis of patients with BC (3). Previous studies have revealed that more than $90 \%$ of human genome sequences do not encode protein. The majority of these sequences are called non-coding RNAs (ncRNAs). microRNAs (miRNAs) are one type of small nc-RNAs (4) and play a role in gene regulation and biological function in numerous cancers. The levels of miRNAs always change along with cancer progression. By binding to the target 3'-untranslated region (3'-UTR), a miRNA can induce mRNA degradation or prevent protein translation (5). miRNAs aberrantly expressed in $\mathrm{BC}$ may play a significant role in cancer metastasis and oncogenesis. Ma et al (6) revealed that miR-148a was an independent prognostic marker of $\mathrm{BC}$, and patients with low miR-148a expression were more likely to have high-grade tumors. Liu et al (7) revealed that miR-101-3p inhibited proliferation and metastasis of BC cells by downregulating EZH2. miR-26a-5p and miR-26b-5p are deemed cancer-suppressive miRNAs, and both have been revealed to be significantly downregulated in multiple cancers $(6,8)$. Additionally, they have been demonstrated to inhibit the proliferation of $\mathrm{BC}$ cells (9). However, the underlying inhibitory mechanisms 
still remain largely unclear. The objective of our study was to investigate the function of miR-26-5p as a tumor suppressor and identify its target genes in BC. Thus, discovering the key miRNAs or elucidating miRNA-regulated mechanisms can provide novel insight and further BC research.

Programmed Cell Death 10 (PDCD10), which has also been called cerebral cavernous malformation 3 (CCM3), was initially referred to as TFAR15 (TF-1 cell apoptosis-related gene). Earlier studies of PDCD10 focused on neural areas. A number of studies have demonstrated that PDCD10 acts as a critical regulator of neuronal survival and integrity $(10,11)$. Subsequently, increasing evidence has indicated a critical role of PDCD10 in regulating cell death and survival. Zhang et al (12) observed that PDCD10 was upregulated in prostate cancer and may play a role in prostate tumorigenesis. Lauenborg et al demonstrated that PDCD10 enhanced the proliferation of malignant $\mathrm{T}$ cells (13). The mechanism by which PDCD10 regulated T-cell proliferation was largely unclear. Therefore, we hypothesized that miR-26a-5p and miR-26b-5p inhibited BC cell proliferation by binding to PDCD10 mRNA. In the present study, we aimed to elucidate the regulatory relationship between $\mathrm{miR}-26 \mathrm{a}-5 \mathrm{p} / \mathrm{miR}-26 \mathrm{~b}-5 \mathrm{p}$ and PDCD10 in BC cell proliferation.

\section{Materials and methods}

Clinical BC specimens. Fresh BC tissues and corresponding adjacent bladder tissues were collected from the Urology Department of Shanghai General Hospital for 8 months from February to October of 2016. None of the patients (Table I) had undergone radio- or chemotherapeutic treatment before radical cystectomy surgery, and all the clinical information of patients is mentioned in Table I. The present study was approved by the Medical Ethics Committee of Shanghai General Hospital, Shanghai Jiao Tong University and informed consent was obtained before surgery from each patient. All specimens were staged in accordance with the tumor-node-metastasis (TNM) classification enacted by the American Joint Committee on Cancer Union for International Cancer Control enacted by the American Joint Committee on Cancer (AJCC) (14).

Animals and cell lines. The experiments on animals were approved by the Ethics Committee for Animal Experimentation of the Shanghai General Hospital of Shanghai Jiao Tong University and strictly abided by the Institutional Guidelines for Use and Care of Laboratory Animals. Forty male pathogen-free 4-week-old nude mice (weight, $15 \mathrm{~g}$ ) were obtained from Slaccas Animal Laboratory (Shanghai, China). All experimental animals were maintained at a temperature of $27 \pm 1^{\circ} \mathrm{C}$ and a humidity of $50 \pm 5 \%$ under diurnal lighting conditions (12-h light/dark cycle) with access to food and water ad libitum. Cell lines of human BC, T24 and 5637, were obtained from the Typical Culture Preservation Commission Cell Bank of the Chinese Academy of Sciences (Shanghai, China). Cell lines were cultured in RPMI-1640 medium supplemented with $1 \%$ penicillin/streptomycin and 10\% FBS (all from HyClone; GE Healthcare Life Sciences, Logan, UT, USA) and under a humidified atmosphere of 5\% $\mathrm{CO}_{2}$ at $37^{\circ} \mathrm{C}(15)$.
In silico analysis of $m i R-26 a-5 p$ and $m i R-26 b-5 p$ target gene. Candidate target genes of miR-26a-5p and miR-26b-5p were predicted using the TargetScan database release 7.1 (http://www.targetscan.org/vert_71/). Genes with cumulative weighed context++ score $\leq-0.20$ were included.

Transduction of BC cell lines and RNA extraction. The short hairpin RNA (shRNA) and GFP-labeled lentivirus vectors containing the miR-26a-5p/miR-26b-5p mimic lentivirus (LV-miR-26a-5p) and the corresponding control miRNA lentivirus (miR-26a-5p/miR-26b-5p-NC), as well as the PDCD10 overexpression lentivirus, the PDCD10 silencing lentivirus, and the corresponding control lentivirus were obtained from GeneChem (Shanghai, China). Cells were seeded in 6-well plates $\left(5 \times 10^{5}\right.$ cells/well) before transduction. The shRNA transduction was conducted with HiPerFect Transfection Reagent according to the manufacturer's instructions (Qiagen, Manchester, UK). Transduction with the lentiviral vectors was conducted using transduction reagents and $8 \mathrm{mg} / \mathrm{ml}$ Polybrene (Genechem) for $12 \mathrm{~h}$. For viral transduction, cells were transduced with a multiplicity of infection (MOI) of 10,100 or 1,000 (16). PDCD10 overexpression, silencing and the corresponding control stable cell lines were then established, and the efficiency of transduction was confirmed by quantitative real-time reverse transcription PCR (RT-qPCR).

RNA extraction and RT-qPCR. Total RNA from the treated cells, including miRNA, was extracted using RNAsimple Total RNA kit (Tiangen Biotech Co., Ltd., Beijing, China) and reverse transcription (RT) was performed using FastKing RT kit (Tiangen Biotech), following the manufacturer's protocol. miRNA sample RT was performed using a miRcute Plus miRNA First-Strand cDNA Synthesis kit (Tiangen Biotech). All samples were assessed using a Quant One Step RT-qPCR kit (SYBR-Green) (Tiangen Biotech). The thermocycling conditions were: $50^{\circ} \mathrm{C}$ for $30 \mathrm{~min}$ for 1 cycle; $95^{\circ} \mathrm{C}$ for $2 \mathrm{~min}$ for 1 cycle; $94^{\circ} \mathrm{C}, 55^{\circ} \mathrm{C}$ and $68^{\circ} \mathrm{C}$ for $20 \mathrm{sec}$ for 40 cycles. U6 and GAPDH were used for normalization, and the relative expression levels of miRNA and PDCD10 were calculated using the $2^{-\Delta \Delta C q}$ method (17). Both of the miRNA primers used were designed and synthesized by Tiangen Biotech (cat. nos. miR-26a-5p:CD201-0319 201221000000002 and miR-26b-5p:CD201-0321). Primers for GAPDH and PDCD10 were designed and synthesized by Shangon Company (Shanghai, China). The following primers were used: 5'-GGT GAAGGTCGGTGTGAACG-3' (sense) and 5'-CTCGCTCCT GGAAGATGGTG-3' (antisense) for GAPDH; and 5'-GAC CCAAGCTCCGCCTCTCCC-3' (sense) and 5'-GGGAGA GGCGGAGCTTGGGTC-3' (antisense) for PDCD10.

Cell proliferation assays in vitro. Cells were seeded in 96-well plates at $3 \times 10^{3}$ cells/well for proliferation assays after transduction for $48 \mathrm{~h}$. Cell proliferation was determined by Cell Counting Kit-8 (CCK-8) reagent (Sigma-Aldrich; Merck KGaA, Darmstadt, Germany) as previously described (18). CCK-8 is a redox indicator that uses the highly water-soluble WST-8 to produce a water-soluble formazan dye, which generated by the activity of dehydrogenases in cells is directly proportional to the number of living cells. BC cell lines were collected after $72 \mathrm{~h}$ of treatment, and $1 \mathrm{ml}$ of 1X FOXP3 Fix/Perm solution 
Table I. Relationship between miR-26a-5p and miR-26b-5p expression levels and clinicopathological significance in BC tissues.

\begin{tabular}{llcccc}
\hline Characteristics & \multicolumn{1}{c}{ Groups } & No. & $\begin{array}{c}\text { Expression of } \\
\text { miR-26a-5p/U6 }\end{array}$ & P-value & $\begin{array}{c}\text { Expression of } \\
\text { miR-26b-5p/U6 }\end{array}$ \\
\hline Tissue & Cancer tissue & & $0.076 \pm 0.002(20$ pairs $)$ & $<0.001$ & $0.104 \pm 0.002(21$ pairs $)$ \\
& Adjacent tissue & & $0.152 \pm 0.006(20$ pairs $)$ & & $0.156 \pm 0.005(21$ pairs $)$ \\
Age, years & $\leq 60$ & 8 & $0.780 \pm 0.004$ & 0.051 & $0.790 \pm 0.041$ \\
& $>60$ & 12 & $0.393 \pm 0.006$ & & $0.619 \pm 0.088$ \\
Sex & Male & 14 & $0.645 \pm 0.151$ & 0.064 & $0.730 \pm 0.078$ \\
TNM stage & Female & 6 & $0.316 \pm 0.031$ & & $0.597 \pm 0.063$ \\
& I/II & 8 & $0.823 \pm 0.138$ & 0.003 & $0.863 \pm 0.036$ \\
& III/IV & 12 & $0.362 \pm 0.053$ & & $0.570 \pm 0.066$ \\
\hline
\end{tabular}

${ }^{\mathrm{a} C h i-s q u a r e ~ t e s t ; ~}{ }^{\mathrm{b}}$ Ridit analysis. BC, bladder cancer.

(BioLegend, Inc., San Diego, CA, USA) was added to each tube for $20 \mathrm{~min}$. According to the manufacturer's instructions, cells were stained with anti-Ki67 antibodies (1:20; cat. no. 350514; BioLegend, Inc.) for $30 \mathrm{~min}$, and isotype $\mathrm{IgG}$ antibody (1:20; cat. no. 400141; BioLegend, Inc.) was used as a control. Ki67 is a nuclear protein which is necessary for cellular proliferation. The treated samples were analyzed with a FACSCalibur flow cytometer and CellQuest software (version 5.2.1) (both from BD Biosciences, Franklin Lakes, NJ, USA). The mean fluorescence intensity (MFI) of Ki67 was detected with a CBA assay (BD Biosciences). MFI is the mean of the fluorescence intensity in the fluorescence channel. The experiments were performed in triplicate.

Western blot analysis. Treated cells were collected, and lysates were prepared in lysis buffer $(50 \mathrm{mM}$ EDTA, $50 \mathrm{mM} \mathrm{NaCl}$, $1 \%$ Triton X-100) containing a protease inhibitor cocktail (Beyotime Institute of Biotechnology, Haimen, China). The protein determination method is BCA. An equal amount of total cell lysate $(30 \mu \mathrm{g})$ was separated on $12 \%$ SDS-PAGE gels and transferred to PVDF membranes. Following antigen blocking with QuickBlock ${ }^{\mathrm{TM}}$ Blocking Buffer for western blot analysis (P0252; Beyotime Institute of Biotechnology), the membranes were incubated with primary antibodies against PDCD10 (1 $\mu \mathrm{g} / \mathrm{ml} ; 1: 1,000$; cat. no. ab110531; Abcam, Cambridge, $\mathrm{UK})$ and GAPDH $(1 \mu \mathrm{g} / \mathrm{ml} ; 1: 1,000$; cat. no. ab8245; Abcam)/Tubulin (1 $\mu \mathrm{g} / \mathrm{ml} ; 1: 5,000$; cat. no. ab7291; Abcam) overnight. Then, the membranes, which were rinsed with TBST, were incubated with goat anti-rabbit secondary antibody (1:1,000; cat. no. A0208; Beyotime Institute of Biotechnology) and visualized with chemiluminescence (New England Nuclear, Boston, MA, USA) by Image Lab software (Bio-Rad Laboratories, Hercules, CA, USA). The experiments were performed in triplicate.

Immunohistochemistry (IHC). Tumor specimens and paired adjacent tissues from bladder patients $(n=10)$ were steeped in $4 \%$ paraformaldehyde and embedded in paraffin $(5 \mu \mathrm{m})$. After heat-induced epitope retrieval, immunohistochemical staining was performed using an avidin-biotin complex method. Subsequently, the endogenous peroxidase activity was blocked by incubation in $3 \% \mathrm{H}_{2} \mathrm{O}_{2}$ for $15 \mathrm{~min}$ and the samples were incubated at $4{ }^{\circ} \mathrm{C}$ overnight with primary anti-PDCD10 antibodies $(10 \mu \mathrm{g} / \mathrm{ml} ; 1: 100$; cat. no. ab110531; Abcam) or a goat IgG isotype in a humid chamber. Subsequently, on the following day, the samples were treated with peroxidase-conjugated anti-rabbit IgG antibody (1:1,000; cat. no. 101ES60; Golden Bridge International, Shanghai, China) for $30 \mathrm{~min}$. Until brown granules appeared, samples were incubated with diaminobenzidine (DAB). Hematoxylin was used for nuclear staining. Sections were evaluated blindly by two researchers with an Olympus BX51+DP70 microscope (Olympus Corp., Tokyo, Japan). The experiments were performed in triplicate and repeated three times.

Luciferase reporter assay. T24 and 5637 cells were seeded in a 24-well plate, and then co-transfected with LV-miR-26a-5p or LV-miR-26b-5p, pMiRTarget-PDCD10-3'-UTR or the mutated 3'-UTR of PDCD10 or an empty vector as a control. The relative dual-luciferase activity of cell lysates was normalized to Renilla luciferase activity and examined with a Dual-Luciferase Reporter Assay system (Promega Corp., Madison, WI, USA). The experiments were performed in triplicate.

Xenograft mouse model. The 5637 cells $\left(1 \times 10^{7}\right)$ stably expressing LV-miR-26a-5p or LV-miR-26b-5p, LV-PDCD10, LV-PDCD10+miR-26a-5p or LV-PDCD10+miR-26b-5p were subcutaneously injected into the left flank area of 4-week-old nude mice ( $\mathrm{n}=6 \mathrm{mice} / \mathrm{group}$ ). Tumor volumes were determined every 3 days $\left(0.5 \mathrm{x}\right.$ length $\mathrm{x}$ width $\left.^{2}\right)$. Three weeks later, the mice were sacrificed by $\mathrm{CO}_{2}$ euthanasia and xenografts were assessed and weighed.

Survival analysis. Clinical data were downloaded by $\mathrm{R} /$ Bioconductor package TCGABiolinks from the TCGA database (https://cancergenome.nih.gov/). Genes or miRNA were excluded if the expression quantity level in $>50 \%$ of the samples was 0. From the entire dataset, 29,627 genes and 627 miRNAs were brought into the study. The miRNA survival analysis brought in 408 samples and the gene survival analysis brought in 409 samples because both sets contained complete clinical data. 
Statistical analysis. All data are presented as the mean \pm SEM and were analyzed using ANOVA, chi-square test and Student's t-test with SPSS 21.0 software (IBM Corp., Armonk, NY, USA). Tukey's test was used to test all pairs of columns. A log-rank test was performed and Kaplan-Meier survival curves were plotted. The P-values were two-sided and a value of $<0.05$ was considered to indicate a statistically significant difference.

\section{Results}

miR-26a-5p and miR-26b-5p inhibit the proliferation of $T 24$ and 5637 cells in vitro. In BC tissues, the expression levels of miR-26a-5p $(n=20)$ and miR-26b-5p $(n=21)$ were significantly reduced compared with those in adjacent bladder tissues based on RT-qPCR results (Fig. 1A). Instead of age and sex, TNM stage was significantly and negatively associated with the levels of miR-26-5p (Table I).

To evaluate the role of miR-26a-5p and miR-26b-5p in BC cell proliferation, we transfected T24 and 5637 cells with miR-26a-5p or miR-26b-5p overexpression lentivirus and a high-efficiency of interference was verified by RT-qPCR (Fig. 1B). CCK-8 assays revealed that, compared with the paired mock or miR-control groups, transduction with miR-26a-5p and miR-26b-5p inhibited proliferation in T24 and 5637 cell lines (Fig. 1C). In addition, flow cytometric assays demonstrated that the MFI of Ki67 was significantly reduced in miR-26a-5p and miR-26b-5p lentivirus-transfected cells compared to mock or miR-control groups (Fig. 1D). These results indicated that miR-26a-5p and miR-26b-5p inhibited cell proliferation in $\mathrm{BC}$ cell lines.

PDCD10 is directly modulated by miR-26a-5p/miR-26b-5p as a target in $B C$ cells. To identify the possible target gene of miR-26-5p in BC, bioinformatics prediction software was used to analyze additional information regarding the proliferation-related molecular mechanisms that are simultaneously regulated by miR-26a-5p and miR-26b-5p in BC cells. miR-26-5p-regulated candidate genes were identified using TargetScan database release 7.1 (http://www.targetscan.org/vert_71/). As displayed in Fig. 2, PDCD10 is the conjunct gene in the intersection of miR-26a-5p-regulated candidate genes and miR-26b-5p-regulated candidate genes (Fig. 2A and B). RT-qPCR and western blot assays revealed that lentivirus-miR-26a-5p or lentivirus-miR-26b-5p stable BC cell lines expressed a lower level of PDCD10 (Fig. 2C and D).

Multiple sequence comparison analysis indicated that there were two possible miR-26a/b-5p binding sites at positions 321-327 and 411-418 in the PDCD10 3'-UTR sequence (Fig. 3A). Thus, in T24 and 5637 cells, dual-luciferase reporter assays were performed to identify whether PDCD10 was directly regulated by miR-26a-5p or miR-26b-5p. We used vectors encoding the partial 3'-UTR sequence of PDCD10, including the potential binding miR-26a-5p/miR-26b-5p site. Following co-transduction with miR-26a-5p/miR-26b-5p and the vector carrying the wild-type sequence containing the PDCD10 3'-UTR with residues 321-327, the luminescence intensity of PDCD10 was significantly reduced (Fig. 3B). However, transduction with the wild-type PDCD10 3'-UTR sequence at positions 411-418 and mutation vector obstructed the decrease in luminescence (Fig. 3C). These data indicated that miR-26a-5p/miR-26b-5p directly bind to specific sites at positions 321-327 of the PDCD10 3'-UTR.

PDCD10 promotes BC cell proliferation in vitro. Immunohistochemical results revealed that $\mathrm{BC}$ tissues expressed a higher level of PDCD10 than paired normal tissues (Fig. 4A and B). We performed loss-of-function studies by transfecting $B C$ cell lines with three sh-PDCD10 plasmids (sh-PDCD10-1, sh-PDCD10-2 and sh-PDCD10-3) in order to explore the function of PDCD10 in BC cell lines and determined that the mRNA and protein levels of PDCD10 in these cell lines were effectively downregulated following transfection (Fig. 4C and D). In addition, transfection with sh-PDCD10 plasmids significantly inhibited proliferation of T24 and 5637 cells (Fig. 4E). In a similar situation, the MFI of Ki67 was significantly lower in T24 and 5637 cells transfected with sh-PDCD10 plasmids than in cells transfected with sh-PDCD10-NC (Fig. 4F). These results ascertained that PDCD10 inhibited the proliferation of $\mathrm{T} 24$ and 5637 cell lines in vitro.

$m i R-26 a-5 p$ and miR-26b-5p inhibit the proliferation-promoting function of PDCD10. In T24 and 5637 cells, the PDCD10 protein levels were evidently higher than in the control group cells following transfection with PDCD10 overexpressing plasmid for $48 \mathrm{~h}$ (Fig. 5A). The high level of PDCD10 promoted proliferation in both T24 and 5637 cells. This effect was partially blocked by the upregulation of the miRNA level by transduction with the corresponding miRNA lentivirus (Fig. 5B). Consistent with these results, the MFI of Ki67 was markedly higher following the upregulation of PDCD10 via transfection with the plasmid, but when the cells were simultaneously transfected with miR-26a-5p or miR-26b-5p lentivirus, the MFI significantly decreased (Fig. 5C). These data indicated that miR-26-5p inhibited the proliferation-stimulating effect in BC cells induced by PDCD10.

PDCD10 promotes growth and progression of $B C$ in vivo and is negatively regulated by $m i R-26 a-5 p$ and $m i R-26 b-5 p$. Having found that miR-26a-5p/miR-26b-5p inhibited the ability of PDCD10 to promote proliferation, we then sought to examine the biological function in vivo. A xenograft mouse model was constructed after LV-miR-26a-5p or LV-miR-26b-5p or mock transfected 5637 cells were subcutaneously injected in nude mice for 7 days. Subsequently, we closely observed the development of tumors and found that miR-26a-5p and miR-26b-5p overexpression xenografts developed much slower than those of the mock group (Fig. 6A-C). To further elucidate the negative regulation of PDCD10 by miR-26a-5p/miR-26b-5p, miRNA-PDCD10 co-overexpression 5637 subcutaneous xenografts were constructed, and they developed more slowly. In addition, growth tendency, anatomic form and weight assays further indicated that both miR-26a-5p and miR-26b-5p inhibited the promoting function of PDCD10 (Fig. 6D-F). As for low and high miR-26-5p and PDCD10 expression groups, Kaplan-Meier survival plots revealed that in patients with high miR-26-5p expression or low PDCD10 expression, overall survival was obviously prolonged compared with patients with low miR-26-5p 

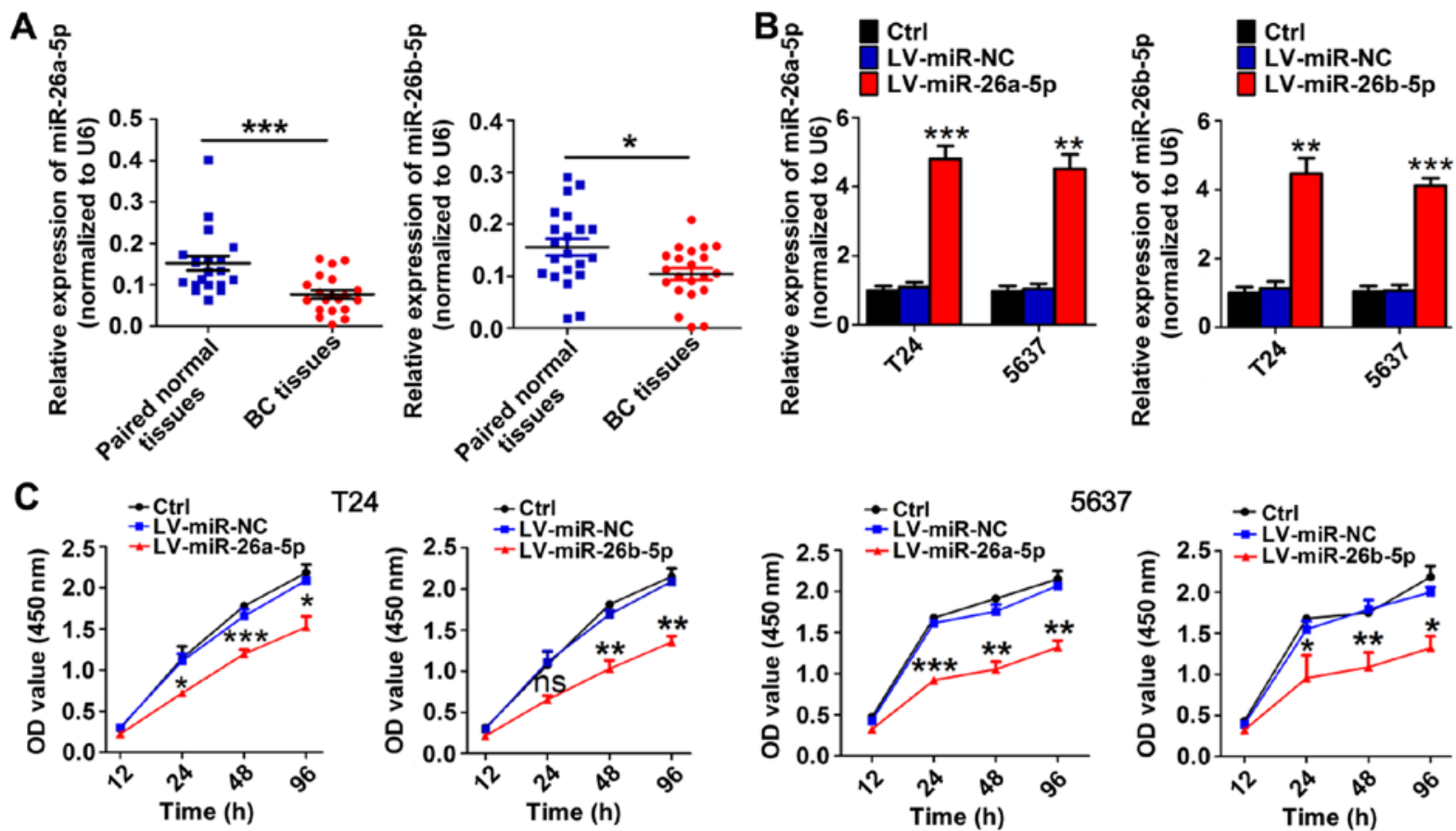

T24
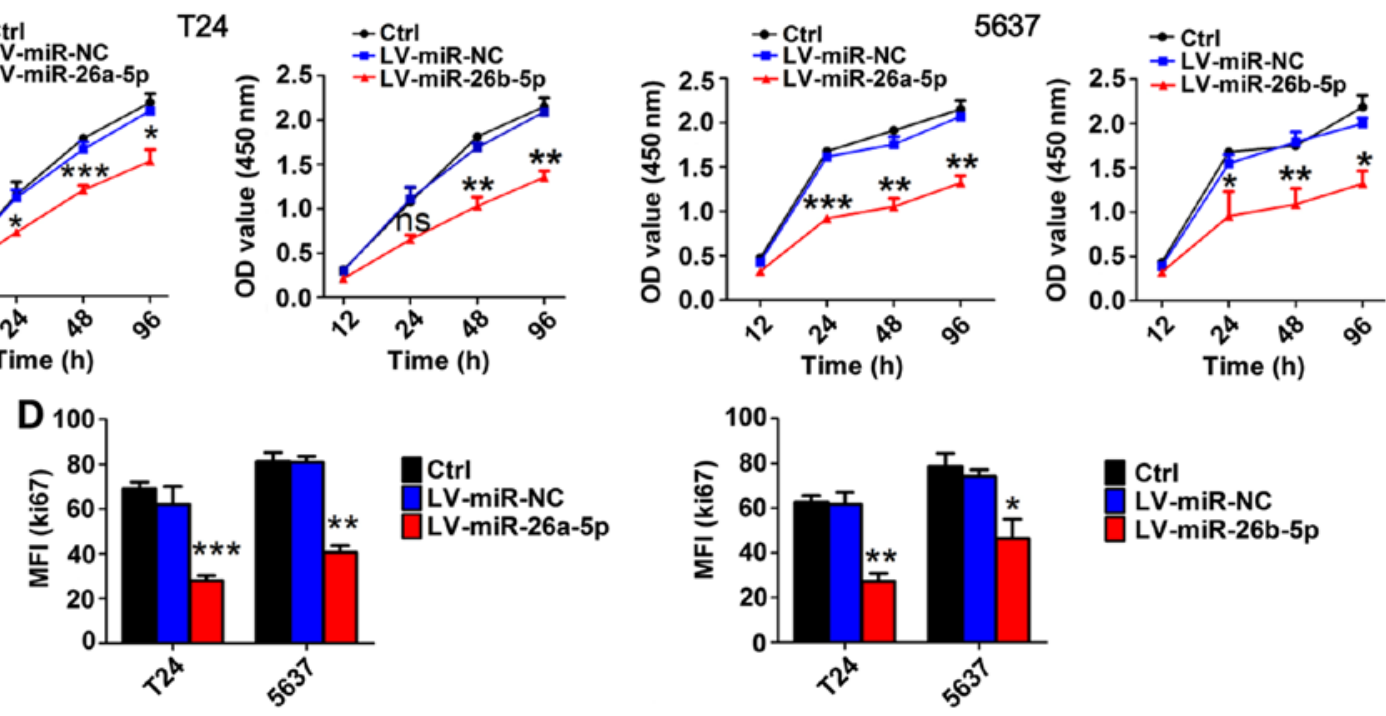

Figure 1. miR-26a-5p and miR-26b-5p inhibit the proliferation of T24 and 5637 cells. (A) The levels of miR-26a-5p (n=20)/miR-26b-5p (n=21) in BC tissues and bladder cancer cell lines (T24 and 5637) compared with paired adjacent bladder tissues based on RT-qPCR. (B) T24 and 5637 were transfected with overexpression lentivirus or miR-control lentivirus. After $48 \mathrm{~h}$, the level of miR-26a-5p and miR-26b-5p was compared with the mock group. (C) CCK-8 assays were used to identify the cell proliferation of miR-26a-5p/26b-5p-transfected BC cells compared with that of mock- or miR-control-transfected BC cells. (D) Following treatment for $48 \mathrm{~h}$, the MFI of Ki67 was tested using flow cytometry in miR-26a-5p/miR-26b-5p-transfected cell lines compared with that of mock- or miR-control-transfected cells. The data are presented as the mean \pm SEM. ${ }^{*} \mathrm{P}<0.05,{ }^{* *} \mathrm{P}<0.01$ or ${ }^{* * * *} \mathrm{P}<0.001$ compared to miR-NC. ns, no statistical significance.

A
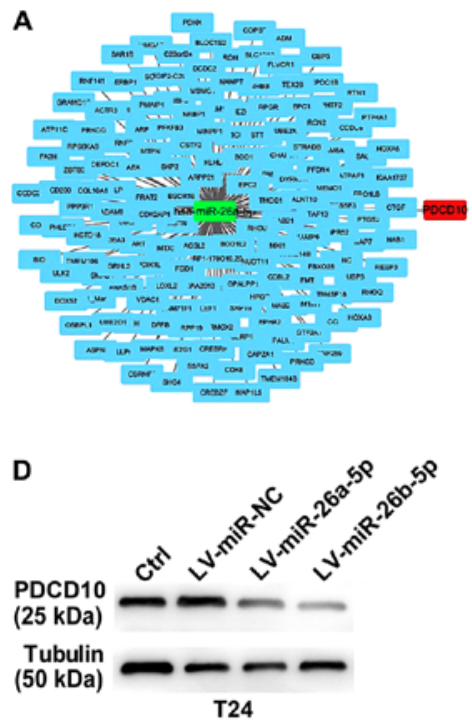

B
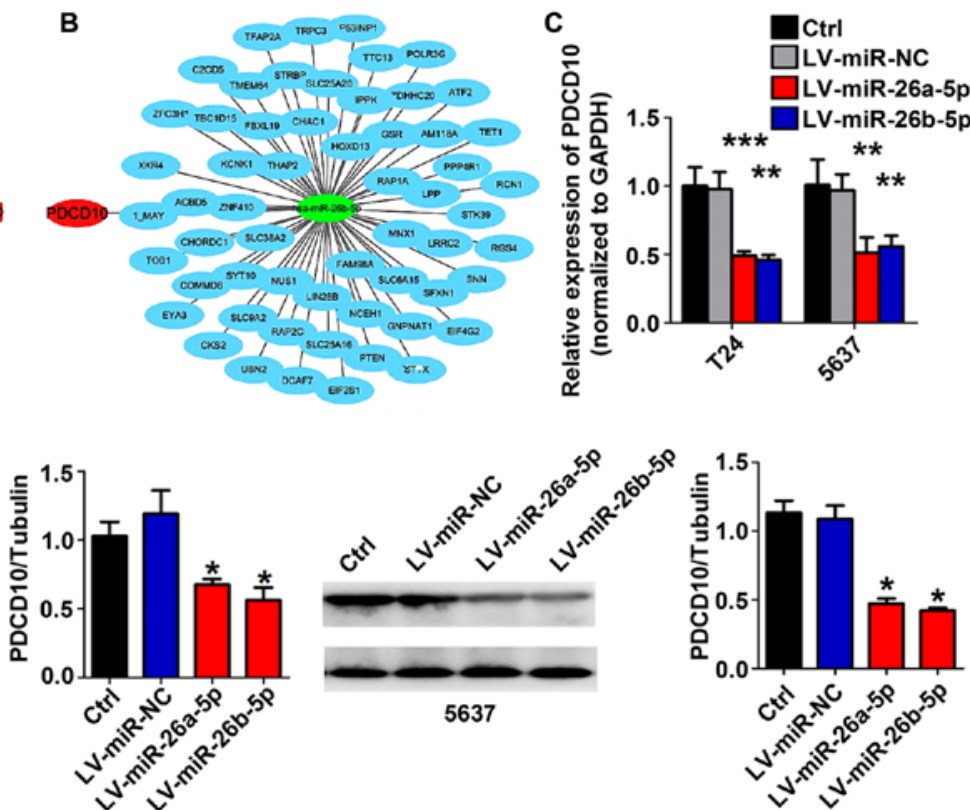
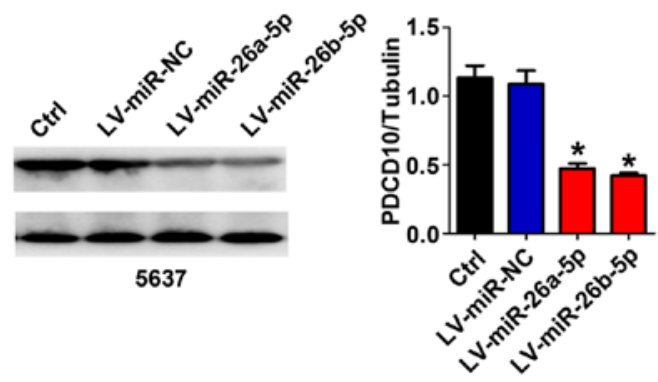

Figure 2. PDCD10 is the target gene of miR-26a-5p/miR-26b-5p in BC cells. (A) Potential target genes of miR-26a-5p and (B) miR-26b-5p were forecasted using a bioinformatics analysis. BC cell lines were transfected with LV-miR-26a-5p/LV-miR-26b-5p or LV-miR-NC, and the RNA and protein levels of the target gene PDCD10 were compared with that of the mock group using (C) RT-qPCR after $48 \mathrm{~h}$ and (D) western blotting after $72 \mathrm{~h} .{ }^{*} \mathrm{P}<0.05,{ }^{* * *} \mathrm{P}<0.01$ or ${ }^{* * * *} \mathrm{P}<0.001$. 
A

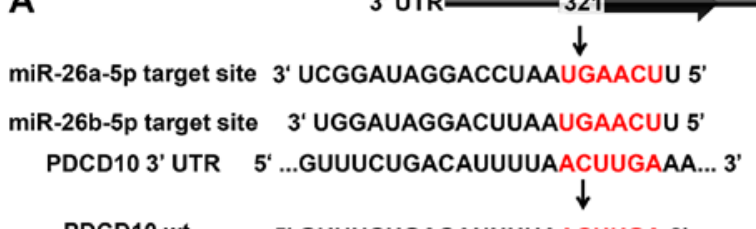

PDCD10 wt 5' GUUUCUGACAUUUUAACUUGA 3' PDCD10 mut 5' GUUUCUGACAUUUUACAGGUC 3'

B
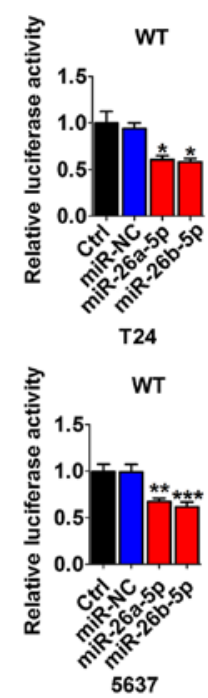
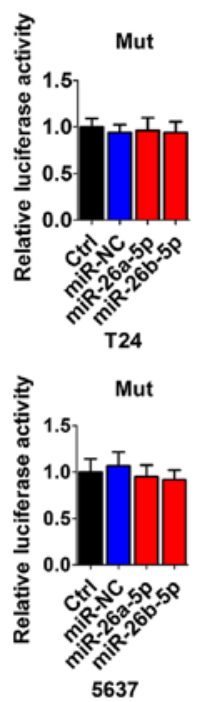

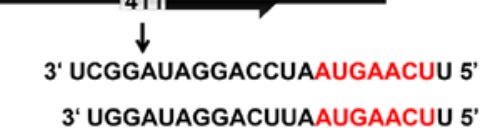

5'...UGUUAAACAUUUCUUUACUUGAA... 3' Human

$\downarrow$

5' GUUUCUgacAUUUUUACUUGA 3'

5' GUUUCUGACAUUUUGCAGgUC 3'

C
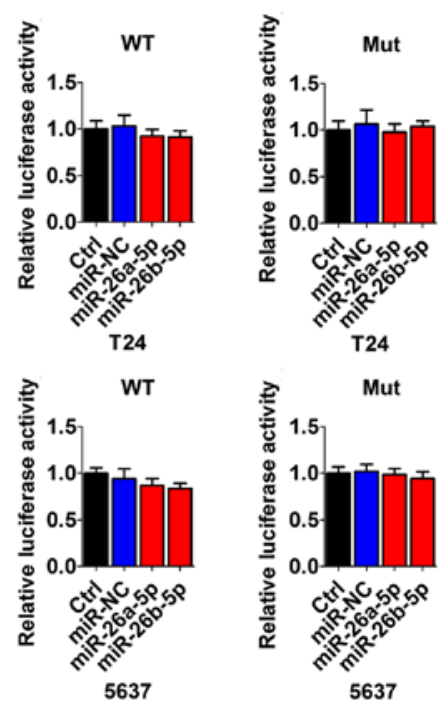

Figure 3. miR-26a-5p/miR-26b-5p directly bind to the PDCD10 3'-UTR at positions 321-327. (A) miR-26a-5p/26b-5p binding sites in the 3'-UTR region of PDCD10 at positions 321-327 and 411-418 were identified by computerized analysis, and a mutation sequence was designed. (B) Following co-transduction with miR-26a-5p/miR-26b-5p and the vector carrying the wild-type 321-327 sequence in the 3'-UTR of PDCD10 mRNA (48 h), Renilla/firefly luciferase activities were significantly reduced, (C) whereas transfection with the wild-type sequence for positions 411-418 in the 3'-UTR of PDCD10 mRNA and the mutation vector obstructed the decrease in luminescence $(48 \mathrm{~h})$. The data are expressed as the mean $\pm \mathrm{SEM} .{ }^{*} \mathrm{P}<0.05,{ }^{* * *} \mathrm{P}<0.01$ or ${ }^{* * * *} \mathrm{P}<0.001$.
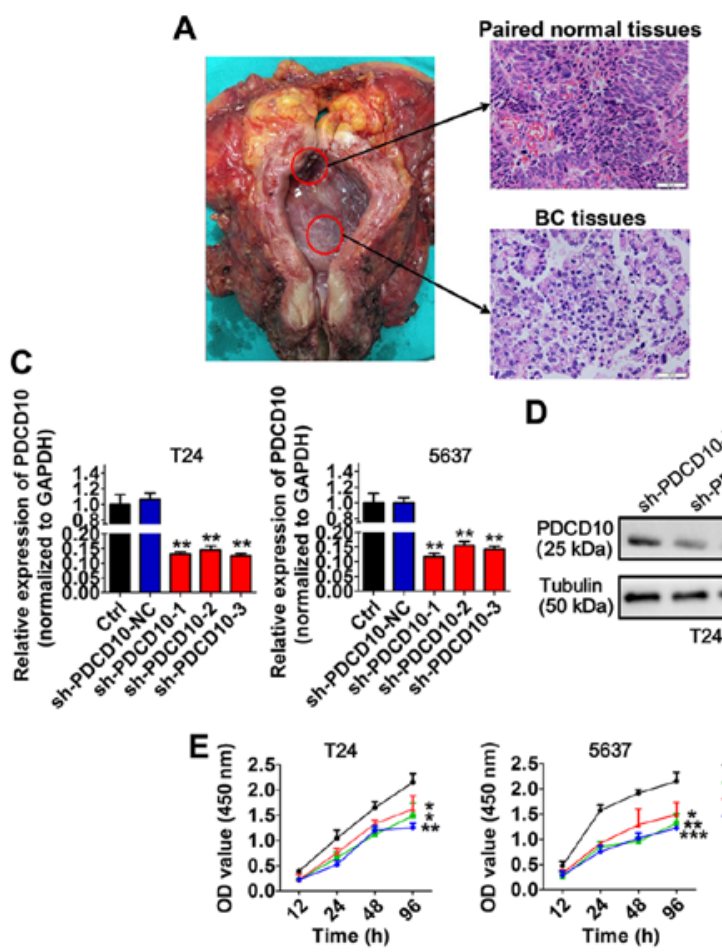

B
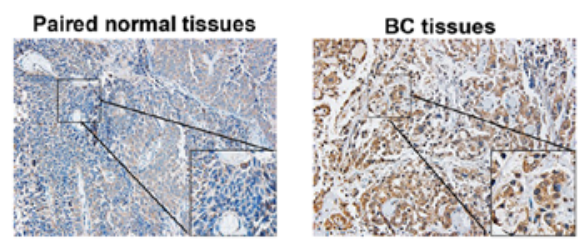

D
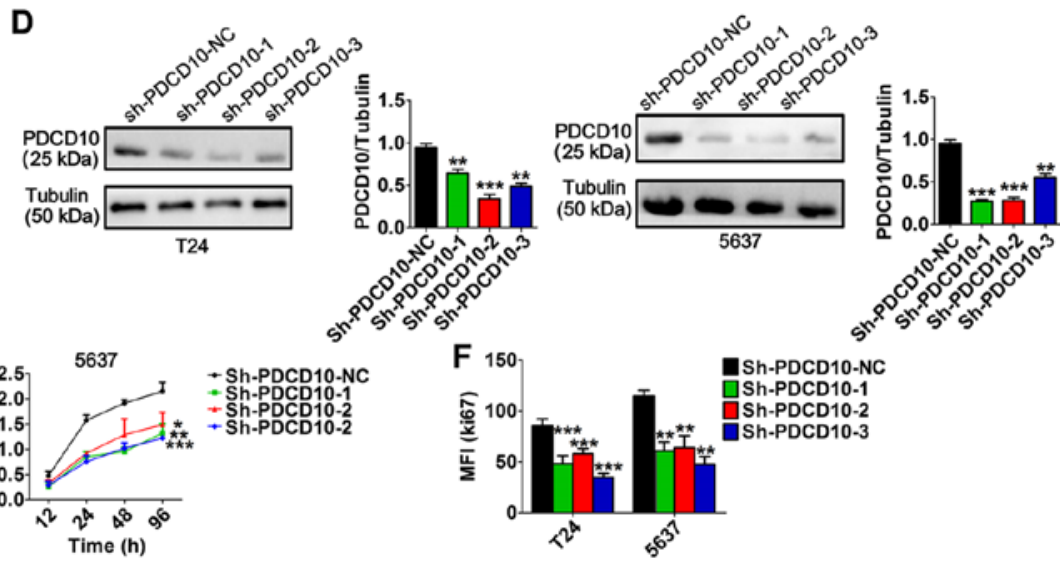

Figure 4. Effects of miR-26a-5p and miR-26b-5p on the proliferation of BC cell lines. (A) The haematoxylin-eosin staining of clinical specimens identified the bladder cancer tissues and paired adjacent bladder tissues. (B) The expression of PDCD10 in bladder cancer tissues and paired adjacent bladder tissues was analyzed using IHC. (C) The knockdown efficiency of sh-PDCD10-1, sh-PDCD10-2 and sh-PDCD10-3 following transduction of T24 and 5637 cells (48 h) was analyzed with RT-qPCR. (D) PDCD10 protein expression was effectively downregulated in both cell lines (72 h) by transduction with the shRNAs according to western blot analyses. (E) The proliferation of sh-PDCD10-transfected cell lines and mock- or miR-control-transfected cells was detected with CCK-8 assays at different time-points (12, 24, 48 and 96 h). (F) The MFI of Ki67 in sh-PDCD10-transfected cell lines was compared with that in mock- or miR-control-transfected cells. The data are expressed as the mean $\pm \mathrm{SEM}$. ${ }^{*} \mathrm{P}<0.05,{ }^{* *} \mathrm{P}<0.01$ or ${ }^{* * *} \mathrm{P}<0.001$ compared to miR-NC. 
A
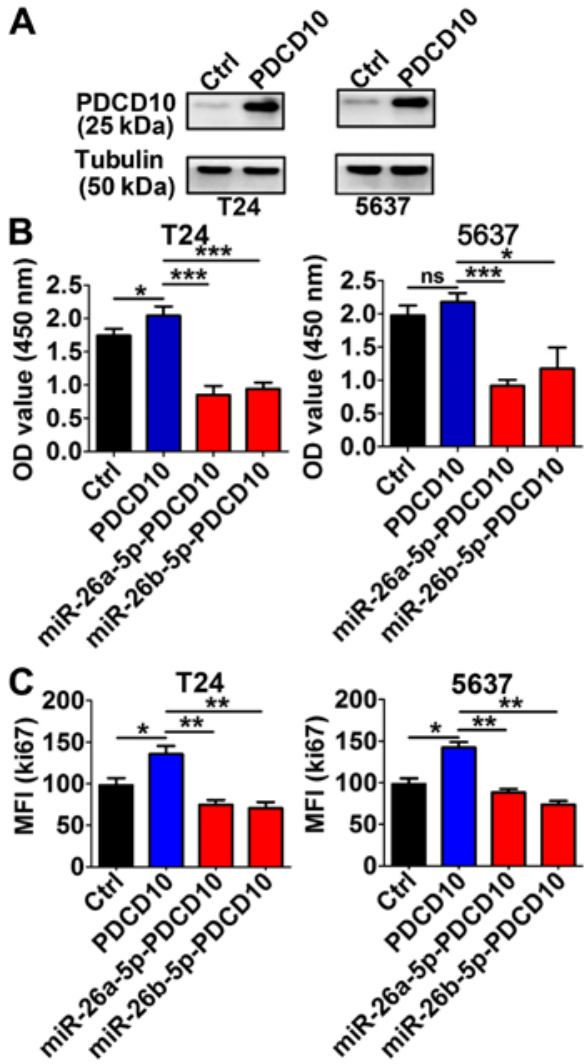

Figure 5. miR-26a-5p and miR-26b-5p inhibit the cancer-promoting function of PDCD10. (A) Western blot assays were used to determine whether overexpression of PDCD10 by sh-RNA could effectively upregulate protein expression in both T24 and 5637 cells (72 h). (B) CCK-8 assays demonstrated that overexpression of PDCD10 could promote proliferation of BC cells, and that proliferation could be inhibited by transduction with LV-miR-26a-5p or LV-miR-26b-5p to simultaneously upregulate mRNA expression $(12,24,48$ and $96 \mathrm{~h}$ ). (C) The MFI density of Ki67 was assessed using flow cytometry and indicated that transduction with PDCD10 overexpression lentivirus and LV-miR-26a-5p or LV-miR-26b-5p to simultaneously upregulate miRNA expression resulted in the downregulation of Ki67 expression $(48 \mathrm{~h})$. The data are expressed as the mean \pm SEM. ${ }^{*} \mathrm{P}<0.05,{ }^{* *} \mathrm{P}<0.01$ or ${ }^{* * *} \mathrm{P}<0.001$ compared to the control group.

expression or high PDCD10 expression (Fig. 6G-H). Animal experiments and clinical data analysis revealed that regulation between miR-26a-5p/miR-26b-5p and PDCD10 had clinical value for evaluating the progression and prognosis of $\mathrm{BC}$ patients.

\section{Discussion}

miRNAs are transcribed to generate long primary transcripts (pri-miRNAs) which are then processed into structures of 60 to $110 \mathrm{nt}$ hairpin precursor miRNAs (pre-miRNAs). These pre-miRNAs are then cleaved by another RNase III enzyme, Dicer, to generate 22 nt miRNAs. Several researchers have reported that there are many miRNAs that play a part in regulating the progression of cancer. Yang et al (19) reported that miR-138-5p is a tumor suppressor that inhibits cell proliferation and invasion by targeting Survivin. In prostate cancer, papillary thyroid carcinoma, and head and neck carcinoma, both miR-26a-5p and miR-26b-5p could inhibit tumor progression (20-22). miR-26a-5p and miR-26b-5p belong to the miR-26 family, and in cancer cell lines, these miRNAs can synergistically block G1/S phase transition. Tian et al (23) reported that both miR-26a-5p and miR-26b-5p play an important role in invasion, proliferation and migration abilities of BC cells. In addition, miR-26-5p plays a key role in cellular growth, development and activation (24). The difference between miR-26a and miR-26b is just several bases. Actually, both of them belong to the miR-26 family and have a similar function by targeting the same mRNAs. The miR-26 family also includes miR-26a-3p and miR-26b-3p. Wang et al (8) reported that miR-26b-3p regulated stem cell proliferation by targeting estrogen receptors. However, the function of miR-26a-3p and miR-26b-3p warrants further exploration. In the present study, we investigated whether miR-26-5p is aberrantly expressed in $\mathrm{BC}$ by collecting paired clinical bladder samples, which constitute a set of paired samples that were perfect for cross-comparison, from 20 patients who underwent radical cystectomy. Similarly, in BC tissues, we observed that the expression level of both miR-26a-5p and miR-26b-5p was significantly lower than in paired normal tissues. Notably, the trend of the majority of our results was similar to Miyamoto et al (9), but they found that aberrant expression of miR-26a-5p did not obviously impact T24 proliferation. However, unlike the previous study, this study included transduction with a high-quality lentivirus to disturb the expression levels of miR-26-5p, which could guarantee a lasting effect. In addition, CCK-8 assays combined with detection of Ki67 using flow cytometry provided stronger and more reasonable results. By analyzing clinical data from TargetScan 7.1 database, we found that patients with high expression of miR-26-5p tend to have a better prognosis.

Upregulation of miR-26-5p appears to be an efficient way to treat $\mathrm{BC}$ but the regulatory network of miRNAs is complicated. An active RNA-induced silencing complex (RISC) includes mature miRNA, containing Dicer and many associated proteins. Certainly, coordinated action between Dicer and other proteins is an efficient method of discarding pre-miRNA (25). Mounting evidence suggests that lncRNAs can function as miRNA sponges and then compete with microRNA for a binding site on protein-coding transcripts. Liu et al revealed that lncRNA SPRY4-IT1 could efficiently reverse the suppression of Enhancer of zeste homolog 2 (EZH2) by directly interacting with miR-101-3p, and this 'sponge' function could promote proliferation and metastasis of BC cells (7). Circular RNA (circRNA) is a new type of ncRNA. Although the roles of circRNAs remain a mystery, insights into circRNAs have revealed that several circRNAs can function as microRNA sponges (26). In addition, Cui et al (27) revealed that the nuclear factor- $\kappa-\mathrm{B}(\mathrm{NF}-\kappa \mathrm{B})$ signaling pathway could directly regulate transcription of miR-130b. Thus, the upstream regulation mechanism of miR-26-5p warrants further study.

Gene silencing may occur either by inhibiting mRNA translation or mRNA degradation. RISC mediates the translation inhibition and degradation of the target mRNA by binding to the 3'-UTR in the target mRNA (28). We used software to assess the intersection of miR-26a-5p and miR-26a-5p candidate target genes, which includes PDCD10. The upstream regulatory mechanism of PDCD10 remains unclear at present. $\mathrm{Xu}$ et al (29) found that miR-181b enhanced angiogenesis of retinoblastoma cells by targeting PDCD10. Wu et al (30) 

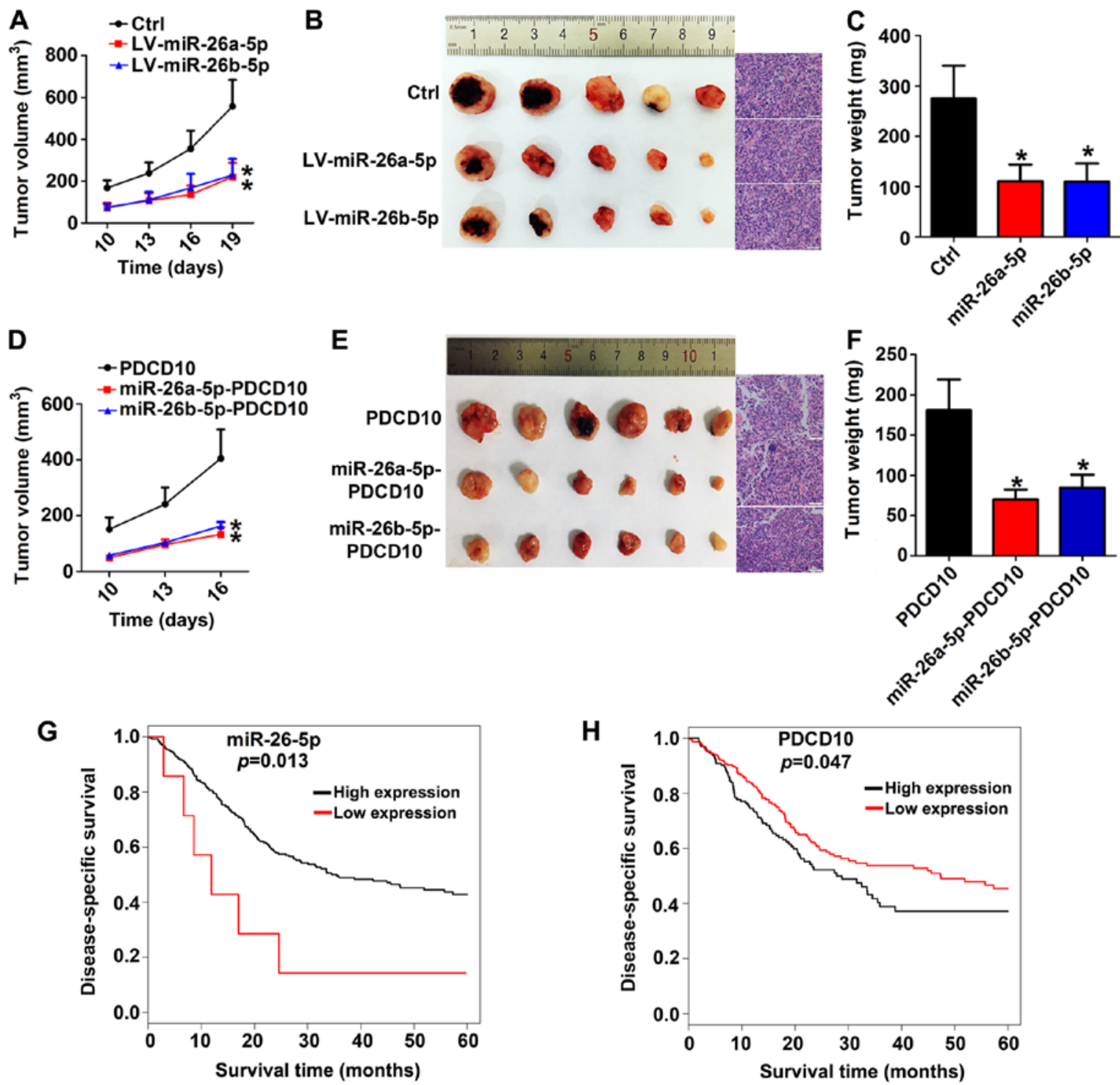

Figure 6. The proliferation-promoting function of PDCD10 is negatively regulated by miR-26a-5p/miR-26b-5p in vivo. (A) Following tumorigenesis, we evaluated tumor progression every 3 days in each group of six nude mice. Xenografts formed from 5637 cells transfected with miR-26a-5p or miR-26b-5p overexpression lentivirus grew less rapidly (10,13,16 and 19 days), and the tumor lesions were (B) smaller and (C) weighed less than the tumors in the mock group. (D) Quantitative assay of tumor volume (10,13,16 and 19 days), (E) presentation of tumor lesions and (F) quantitative assays of tumor weights revealed that mice xenografts formed from cells simultaneously transfected with miRNA overexpression lentivirus and PDCD10 overexpression lentivirus inhibited the tumor-promoting ability of PDCD10. (G and $\mathrm{H}$ ) The Kaplan-Meier survival curve of miR-26-5p and PDCD10 revealed that the prognosis of BC patients with a high level of miR-26-5p and low PDCD10 expression was better than that of patients with a low level of miR-26-5p and high PDCD10 expression. The data are expressed as the mean \pm SEM. ${ }^{*} \mathrm{P}<0.05$ compared to the control group.

demonstrated that miR-613 could regulate cardiomyocyte apoptosis by targeting the PDCD10 gene. These results indicated that PDCD10 can be regulated by miRNA. In addition, there are two potential binding sites in the 3'-UTR of PDCD10 that miR-26a-5p and miR-26a-5p can simultaneously bind. Our results indicated that miR-26a-5p/miR-26b-5p directly bound to specific sites at positions 321-327 of the PDCD10 3 '-UTR. To gain insight into the regulatory relationship between miR-26a/b-5p and PDCD10, we used RT-qPCR and western blotting to demonstrate that $\mathrm{miR}-26 \mathrm{a} / \mathrm{b}-5 \mathrm{p}$ downregulated PDCD10. We also identified the binding site of miRNA using luciferase reporter assays. Furthermore, we demonstrated that upregulation of $\mathrm{miR}-26 \mathrm{a} / \mathrm{b}-5 \mathrm{p}$ weakened the cancer proliferation function of PDCD10 both in vitro and in vivo.
Several studies have reported that PDCD10 is aberrantly expressed in some types of human cancers (31). In this study, BC tissues expressed a higher level of PDCD10 than paired para-carcinoma tissues. PDCD10 is an evolutionarily highly conserved protein that is associated with cell proliferation and apoptosis. Knockdown of PDCD10 by sh-RNA inhibited the proliferation of T24 and 5637 cell lines. This observation was in accordance with previous findings that revealed that silencing PDCD10 could inhibit the proliferation of endothelial cells (32). Lambertz et al (33) proposed that PDCD10 potentially participated in tumor proliferation, apoptosis, hyper-angiogenesis and peritumoral edema in human glioblastoma. In addition, downregulation of PDCD10 inhibited tumor cell proliferation (33). In addition to the effect on proliferation, previous studies indicated a positive correlation between 
PDCD10 and tumor cell apoptosis (33), and RNA interference indicated that PDCD10 regulated cell mitogenesis (34). Synthetic biological functions of PDCD10 warrant more investigation. In contrast to the well-established mechanism of PDCD10 in vessel permeability and neo-angiogenesis, limited information about PDCD10 in cancer research has been reported $(35,36)$. He et al $(37)$ forecasted that PDCD10 may play an important role in cancer since it can influence angiogenesis by targeting vascular endothelial growth factor receptor 2 (VEGFR2) (37). He et al revealed that PDCD10 deletion reduced VEGFR2 signaling in embryonic and endothelial cells (37). Notably, VEGF/VEGFR2 signaling could regulate germ cell proliferation in vitro and promote mouse testicular regeneration in vivo (38). This indicated that PDCD10 may regulate proliferation of BC possibly through VEGFR2 signaling. Ma et al (39) revealed that PDCD10 interacted with the kinase MST4 and promoted cell growth and transformation through the extracellular signal-regulated kinase (ERK) pathway. Riverso et al (40) reported that Krüppel-like factor 4 (KLF4) is regulated by ERK signaling and promoted melanoma cell growth. Through bioinformatics analysis, clinical data also indicated that prognosis of patients with low expression of PDCD10 was better than that of patients with high PDCD10 expression.

Further studies on PDCD10 downstream signaling are necessary. Lauenborg et al (13) reported that PDCD10 regulated proliferation and survival of malignant $T$ cells by activating Jak3 signaling. In addition, PDCD10 could directly bind to the phosphatase domain of Fas-associated phosphatase-1 (FAP-1) and serine/threonine kinase 25 (STK25) in cerebral cavernous malformations (41). Furthermore, PDCD10 could form a complex with GM130, a Golgi-resident protein, and the germinal center kinase III (GCKIII) family to regulate cell migration (42). The effect of PDCD10 may be the result of a modulation of one or more of these processes in our experiments, which warrants further study.

In conclusion, miR-26a-5p and miR-26b-5p play an antitumor role in BC, and PDCD10 is an important downstream target gene of miR-26a-5p/miR-26b-5p. Considering that patients with a low PDCD10 expression level in BC tissues appear to have a better prognosis than patients with high PDCD10 expression, PDCD10 may be a new diagnostic biomarker in BC, and the miR-26a-5p/miR-26b-5p/PDCD10 axis may be a new therapeutic target.

\section{Acknowledgements}

Not applicable.

\section{Funding}

The present study was supported by the Natural Science Foundation of China (no. 81672515), the Shanghai Pujiang Program (no. 16PJD039) and the Commission of Gaofeng Clinical Medicine Grant (no. 20172019).

\section{Availability of data and materials}

All data generated or analyzed during this study are included in this published article.

\section{Authors' contributions}

ZHL and MQL designed this research and provided research direction. KW and XYM performed the research and collected the data. JTJ, MYT, RJW and WJZ analyzed the data and finished the animal experiments. XW and YYH drafted the manuscript and revised it critically for important content. All authors read and approved the manuscript and agree to be accountable for all aspects of the research in ensuring that the accuracy or integrity of any part of the work are appropriately investigated and resolved.

\section{Ethics approval and consent to participate}

The present study was approved by the Medical Ethics Committee of Shanghai General Hospital, Shanghai Jiao Tong University and informed consent was obtained before surgery from each patient. The animal experiments were approved by the Ethics Committee for Animal Experimentation of the Shanghai General Hospital of Shanghai Jiao Tong University and strictly abided by the Institutional Guidelines for Use and Care of Laboratory Animals.

\section{Patient consent for publication}

Not applicable.

\section{Competing interests}

We declare that none of the authors have any financial and personal relationships with other people or organizations that can inappropriately influence the quality of the present study. The authors declare that they have no competing interests.

\section{References}

1. Siegel RL, Miller KD and Jemal A: Cancer statistics, 2017. CA Cancer J Clin 66: 7-30, 2016.

2. Barton MK: High morbidity and mortality found for high-risk, non-muscle-invasive bladder cancer. CA Cancer J Clin 63: 371-372, 2013.

3. Comprehensive molecular characterization of urothelial bladder carcinoma. Nature 507: 315-322, 2014.

4. Bartel DP: MicroRNAs: Target recognition and regulatory functions. Cell 136: 215-233, 2009.

5. Bartel DP: MicroRNAs: Genomics, biogenesis, mechanism, and function. Cell 116: 281-297, 2004.

6. Ma L, Xu Z, Xu C and Jiang X: MicroRNA-148a represents an independent prognostic marker in bladder cancer. Tumour Biol 37: 7915-7920, 2016.

7. Liu D, Li Y, Luo G, Xiao X, Tao D, Wu X, Wang M, Huang C, Wang L, Zeng F, et al: LncRNA SPRY4-IT1 sponges miR-101-3p to promote proliferation and metastasis of bladder cancer cells through up-regulating EZH2. Cancer Lett 388: 281-291, 2017.

8. Wang Y, Sun B, Zhao X, Zhao N, Sun R, Zhu D, Zhang Y, Li Y, $\mathrm{Gu} \mathrm{Q}$, Dong X, et al: Twist1-related miR-26b-5p suppresses epithelial-mesenchymal transition, migration and invasion by targeting SMAD1 in hepatocellular carcinoma. Oncotarget 7 : 24383-24401, 2016.

9. Miyamoto K, Seki N, Matsushita R, Yonemori M, Yoshino H, Nakagawa $M$ and Enokida H: Tumour-suppressive miRNA-26a-5p and $m i R-26 b-5 p$ inhibit cell aggressiveness by regulating PLOD2 in bladder cancer. Br J Cancer 115: 354-363, 2016.

10. Lin C, Meng S, Zhu T and Wang X: PDCD10/CCM3 acts downstream of \{gamma\}-protocadherins to regulate neuronal survival. J Biol Chem 285: 41675-41685, 2010. 
11. Stamatovic SM, Sladojevic N, Keep RF and Andjelkovic AV: PDCD10 (CCM3) regulates brain endothelial barrier integrity in cerebral cavernous malformation type 3: Role of CCM3-ERK1/2-cortactin cross-talk. Acta Neuropathol 130: $731-750,2015$.

12. Zhang H, Ma X, Peng S, Nan X and Zhao H: Differential expression of MST4, STK 25 and PDCD10 between benign prostatic hyperplasia and prostate cancer. Int J Clin Exp Pathol 7: 8105-8111, 2014.

13. Lauenborg B, Kopp K, Krejsgaard T, Eriksen KW, Geisler C, Dabelsteen S, Gniadecki R, Zhang Q, Wasik MA, Woetmann A, et al: Programmed cell death-10 enhances proliferation and protects malignant $\mathrm{T}$ cells from apoptosis APMIS 118: 719-728, 2010.

14. Sobin LH and Compton CC: TNM seventh edition: What's new, what's changed: Communication from the international union against cancer and the American Joint Committee on Cancer. Cancer 116: 5336-5339, 2010.

15. Cui X, Shen D, Kong C, Zhang Z, Zeng Y, Lin X and Liu X: $\mathrm{NF}-\kappa \mathrm{B}$ suppresses apoptosis and promotes bladder cancer cell proliferation by upregulating survivin expression in vitro and in vivo. Sci Rep 7: 40723, 2017.

16. Sudarshan S, Holman DH, Hyer ML, Voelkel-Johnson C, Dong JY and Norris JS: In vitro efficacy of Fas ligand gene therapy for the treatment of bladder cancer. Cancer Gene Ther 12: 12-18, 2005.

17. Livak KJ and Schmittgen TD: Analysis of relative gene expression data using real-time quantitative PCR and the $2^{-\Delta \Delta C_{\mathrm{T}}}$ method. Methods 25: 402-408, 2001.

18. Lin H, Liu W, Fang Z, Liang X, Li J, Bai Y, Lin L, You H, Pei Y, Wang F, et al: Overexpression of DHX32 contributes to the growth and metastasis of colorectal cancer. Sci Rep 5: 9247, 2015.

19. Yang R, Liu M, Liang H, Guo S, Guo X, Yuan M, Lian H, Yan X, Zhang S, Chen X, et al: $m i R-138-5 p$ contributes to cell proliferation and invasion by targeting Survivin in bladder cancer cells. Mol Cancer 15: 82, 2016.

20. Kato M, Goto Y, Matsushita R, Kurozumi A, Fukumoto I, Nishikawa R, Sakamoto S, Enokida H, Nakagawa M, Ichikawa T, et al: MicroRNA-26a/b directly regulate La-related protein 1 and inhibit cancer cell invasion in prostate cancer. Int J Oncol 47: 710-718, 2015.

21. Fu X, Meng Z, Liang W, Tian Y, Wang X, Han W, Lou G, Wang X, Lou F, Yen Y, et al: miR-26a enhances miRNA biogenesis by targeting Lin28B and Zcchc11 to suppress tumor growth and metastasis. Oncogene 33: 4296-4306, 2014.

22. Fukumoto I, Kikkawa N, Matsushita R, Kato M, Kurozumi A, Nishikawa R, Goto Y, Koshizuka K, Hanazawa T,Enokida H, et al: Tumor-suppressive microRNAs (miR-26a/b, miR-29a/b/c and miR-218) concertedly suppressed metastasis-promoting LOXL2 in head and neck squamous cell carcinoma. J Hum Genet 61: 109-118, 2016

23. Tian L, Fang YX, Xue JL and Chen JZ: Four microRNAs promote prostate cell proliferation with regulation of PTEN and its downstream signals in vitro. PLoS One 8: e75885, 2013.

24. Icli B, Dorbala P and Feinberg MW: An emerging role for the miR-26 family in cardiovascular disease. Trends Cardiovasc Med 24: 241-248, 2014

25. Fareh M, Yeom KH, Haagsma AC, Chauhan S, Heo I and Joo C: TRBP ensures efficient Dicer processing of precursor microRNA in RNA-crowded environments. Nat Commun 7: 13694, 2016.

26. Meng X, Li X, Zhang P, Wang J, Zhou Y and Chen M: Circular RNA: An emerging key player in RNA world. Brief Bioinform 18: 547-557, 2017.
27. Cui X, Kong C, Zhu Y, Zeng Y, Zhang Z, Liu X, Zhan B, Piao C and Jiang Z: miR-130b, an onco-miRNA in bladder cancer, is directly regulated by $\mathrm{NF}-\kappa \mathrm{B}$ and sustains $\mathrm{NF}-\kappa \mathrm{B}$ activation by decreasing Cylindromatosis expression. Oncotarget 7 : 48547-48561, 2016.

28. Nahvi A, Shoemaker CJ and Green R: An expanded seed sequence definition accounts for full regulation of the hid 3' UTR by bantam miRNA. RNA 15: 814-822, 2009.

29. Xu X, Ge S, Jia R, Zhou Y, Song X, Zhang H and Fan X: Hypoxia-induced miR-181b enhances angiogenesis of retinoblastoma cells by targeting PDCD10 and GATA6. Oncol Rep 33: 2789-2796, 2015.

30. Wu Z, Qi Y, Guo Z, Li P and Zhou D: miR-613 suppresses ischemia-reperfusion-induced cardiomyocyte apoptosis by targeting the programmed cell Death 10 gene. Biosci Trends 10: 251-257, 2016

31. Chen PY, Chang WS, Chou RH, Lai YK, Lin SC, Chi CY and Wu CW: Two non-homologous brain diseases-related genes, SERPINI1 and PDCD10, are tightly linked by an asymmetric bidirectional promoter in an evolutionarily conserved manner. BMC Mol Biol 8: 2, 2007.

32. You C, Sandalcioglu IE, Dammann P, Felbor U, Sure U and Zhu Y: Loss of CCM3 impairs DLL4-Notch signalling: Implication in endothelial angiogenesis and in inherited cerebral cavernous malformations. J Cell Mol Med 17: 407-418, 2013.

33. Lambertz N, El HN, Kreitschmann-Andermahr I, Stein KP, Dammann P, Oezkan N, Mueller O, Sure U and Zhu Y: Downregulation of programmed cell death 10 is associated with tumor cell proliferation, hyperangiogenesis and peritumoral edema in human glioblastoma. BMC Cancer 15: 759, 2015.

34. Kamath RS, Fraser AG, Dong Y, Poulin G, Durbin R, Gotta M, Kanapin A, Le Bot N, Moreno S, Sohrmann M, et al: Systematic functional analysis of the Caenorhabditis elegans genome using RNAi. Nature 421: 231-237, 2003.

35. Stockton RA, Shenkar R, Awad IA and Ginsberg MH: Cerebral cavernous malformations proteins inhibit Rho kinase to stabilize vascular integrity. J Exp Med 207: 881-896, 2010.

36. Zhu Y, Zhao K, Prinz A, Keyvani K, Lambertz N, Kreitschmann-Andermahr I, Lei T and Sure U: Loss of endothelial programmed cell death 10 activates glioblastoma cells and promotes tumor growth. Neuro Oncol 18: 538-548, 2016.

37. He Y, Zhang H, Yu L, Gunel M, Boggon TJ, Chen H and Min W: Stabilization of VEGFR2 signaling by cerebral cavernous malformation 3 is critical for vascular development. Sci Signal 3: ra26, 2010.

38. Tian R, Yang S, Zhu Y, Zou S, Li P, Wang J, Zhu Z, Huang Y, $\mathrm{He} \mathrm{Z}$ and $\mathrm{Li} \mathrm{Z}$ : VEGF/VEGFR ${ }_{2}$ signaling regulates germ cell proliferation in vitro and promotes mouse testicular regeneration in vivo. Cells Tissues Organs 201: 1-13, 2016.

39. Ma X, Zhao H, Shan J, Long F, Chen Y, Chen Y, Zhang Y, Han X and Ma D: PDCD10 interacts with Ste20-related kinase MST4 to promote cell growth and transformation via modulation of the ERK pathway. Mol Biol Cell 18: 1965-1978, 2007.

40. Riverso M, Montagnani V and Stecca B: KLF4 is regulated by $\mathrm{RAS} / \mathrm{RAF} / \mathrm{MEK} / \mathrm{ERK}$ signaling through E2F1 and promotes melanoma cell growth. Oncogene 36: 3322-3333, 2017.

41. Voss K, Stahl S, Schleider E, Ullrich S, Nickel J, Mueller TD and Felbor U: CCM3 interacts with CCM2 indicating common pathogenesis for cerebral cavernous malformations. Neurogenetics 8: 249-256, 2007.

42. Fidalgo M, Fraile M, Pires A, Force T, Pombo C and Zalvide J: CCM3/PDCD10 stabilizes GCKIII proteins to promote Golgi assembly and cell orientation. J Cell Sci 123: 1274-1284, 2010 . 